04

\title{
Разряд с жидким неметаллическим катодом (водопроводная вода) в потоке воздуха атмосферного давления
}

\author{
(C) Ю.А. Баринов, С.М. Школьник
}

Физико-технический институт им. А.Ф. Иоффре, 194021 Санкт-Петербург, Россия

e-mail: yury@mail.ioffe.ru

(Поступило в Редакцию 30 марта 2016 г.)

\begin{abstract}
Представлено экспериментальное исследование разряда с жидким неметаллическим катодом (водопроводная вода) в потоке воздуха атмосферного давления. Данный разряд представляет собой модификацию ранее исследованного разряда с жидкими неметаллическими электродами. Описана конструкция разрядного узла и приведены некоторые результаты исследований.
\end{abstract}

\section{Введение}

В настоящее время большой интерес вызывают разряды, в которых в качестве электродов (одного или обоих) используются неметаллические (слабопроводящие) жидкости и электролиты, в том числе водопроводная или техническая вода $[1,2]$. Такие разряды могут быть реализованы при питании постоянным током как при пониженном, так и при атмосферном давлении в различных газах. Разряд с жидкими неметаллическими электродами (РЖНЭ) генерирует неравновесную плазму с высокой концентрацией активных радикалов [3-5]. Представляет интерес исследование подобного разряда в потоке газа. Плазматроны с жидким катодом и с обоими жидкими электродами ранее уже были разработаны, однако исследования генерируемой ими плазмы не проводились.

На рис. 1 схематично показана верхняя часть разрядного узла. Он состоит из трех коаксиальных вертикально расположенных кварцевых трубок 7. В центральную трубку подается водопроводная вода 9. Вторая трубка используется для сбора воды. Воздух 8 подается между второй и внешней трубками. Внешняя трубка (диаметром $70 \mathrm{~mm}$ ) закрыта фторопластовым фланцем толщиной $6 \mathrm{~mm}$ 6. В центр фланца вставлена кварцевая трубочка 3 длиной $7 \mathrm{~mm}$ с внутренним диаметром $3 \mathrm{~mm}$, формирующая поток газа. Анод 2 (медный штырь диаметром $4 \mathrm{~mm}$ ) закреплен над верхним срезом трубочки на расстоянии $5 \mathrm{~mm}$. Конец штыря смещен от оси разрядного узла на $\sim 1 \mathrm{~mm}$. К водяному катоду ток подводится с помощью никелевой пластины 1. Пластина утоплена в воде на глубину $\sim 5 \mathrm{~mm}$.

Для питания разряда был использован регулируемый источник постоянного тока с выходным напряжением до $6 \mathrm{kV}$. В цепь питания разряда включен балластный резистор $7.5 \mathrm{k} \Omega$. Анод заземлен. Поджиг разряда производился вручную сквозь отверстие во фланце специальным проводником на изолированной рукоятке.

Эксперименты проводились при токах $I=0.1,0.2$ и $0.3 \mathrm{~A}$. Ток измерялся на шунте, а напряжение между металлическими электродами - через делитель $1: 1000$. Для электрических измерений использовался осциллограф Tektronix TDS3012. Для продувки воздуха применялся безмаслянный компрессор УК40-2М. Расход воздуха контролировался с помощью датчика Honeywell AWM720P1. Измерения были выполнены при расходах $Q=5,15$ и $201 / \mathrm{min}$. При токе $I=0.1$ А разряд горел устойчиво только при $Q \leq 51 / \mathrm{min}$. Для устойчивого горения разряда при расходах $Q>51 / \min$ было необходимо увеличивать ток.

Для спектроскопических измерений использовалась оптическая схема, описанная в [4], которая обеспечивала спектральное разрешение $0.2 \mathrm{~nm}$. Для фотографирования разряда использовалась высокоскоростная видеокамера Phantom M310 с макрообъективом Carl Zeiss 100/2. Камера чувствительна в диапазоне спектра 450-800 nm), максимум чувствительности находится в красной области.

Для измерения распределение потенциала плазмы вдоль оси разряда использовался зонд из молибденовой проволоки диаметром $0.3 \mathrm{~mm}$. Длина рабочей части зонда составляла $\sim 1 \mathrm{~mm}$. Воизбежание перегрева зонд

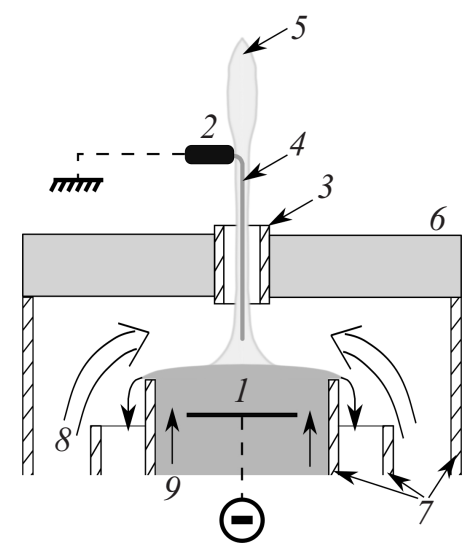

Рис. 1. Верхняя часть разрядного узла: 1 - металлический токоподвод к водяному катоду, 2 - металлический анод, 3 кварцевая трубочка, формирующая поток, 4 - токовый канал, 5 - плазменный бестоковый факел, $6-$ фторопластовый фланец, $7-$ кварцевые трубки, $8-$ подача газа, $9-$ подача и сток воды. 


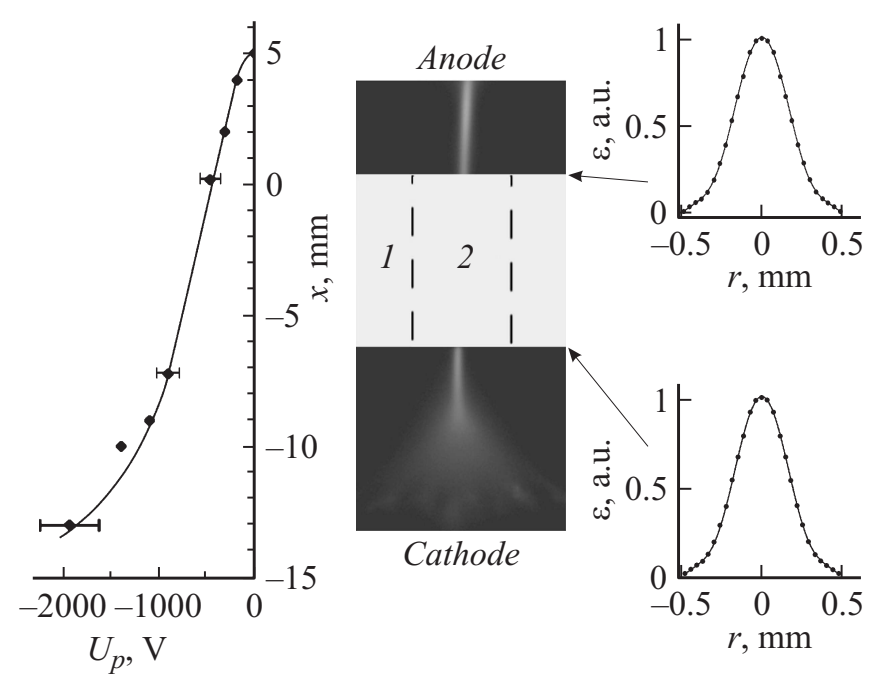

Рис. 2. Совмещенные результаты электрических и оптических измерений: в центре - фотография разряда $(1-$ фланец, 2 - трубочка), слева - распределение потенциала вдоль оси разряда, справа - радиальные распределения коэффициента эмиссии плазмы.

погружался в плазму кратковременно (менее $1 \mathrm{~s}$ ). Измерялся потенциал плавающего зонда, который в рассматриваемых условиях отличается от потенциала плазмы на величину $\sim k T_{e} / e[6]$. Этим отличием мы пренебрегали, поскольку, как будет видно ниже, $k T_{e} / e<1 \mathrm{~V}$. Вопросы, связанные с техникой и методикой зондовых измерений в плазме РЖНЭ при атмосферном давлении, подробно рассмотрены в [7]. Температура газа в бестоковой части потока (над анодом) определялась $\mathrm{W}-\mathrm{Re}$-термопарой из проволоки диаметром $0.3 \mathrm{~mm}$. Термопара была заземлена. Сигнал с термопары поступал на осциллограф. Сразу после того как сигнал с пары переставал меняться, она удалялась из потока, так как при нагревании в воздушной атмосфере $\mathrm{W}$ быстро окисляется и проволока перегорает. Удалось измерить температуры до $2000 \mathrm{~K}$. Измерения удавалось повторить несколько раз, прежде чем термопара разрушалась.

Измерения показали, что ток и напряжение разряда зашумлены. На осциллограммах напряжения наблюдаются низкочастотные периодические колебания с характерной частотой $\sim 5-7 \mathrm{kHz}$. Амплитуда этих колебаний не превышает 3\% от общего напряжения на металлических электродах. Как показали результаты скоростной видеосъемки, эти колебания можно связать с небольшим выносом канала разряда потоком газа выше среза анода с последующим шунтированием образовавшегося изгиба. Среднее значение напряжения зависит от тока и расхода газа. При изменении этих величин в указанных выше пределах оно меняется от $\sim 2.7$ до $\sim 3.1 \mathrm{kV}$, из которых около $1 \mathrm{kV}$ (зависит от тока) падает в воде, покрывающей металлический токоподвод.

На рис. 2 приведены совмещенные результаты электрических и оптических измерений. В центре рисунка расположена фотография разряда. Ось $x$ направлена от катода к аноду. За нуль принята точка на пересечении оси с верхним срезом трубочки. Сама трубочка 2 на фотографии не видна, так как большая ее часть перекрывается фланцем 1. Благодаря малому времени экспозиции $(50 \mu \mathrm{s})$ видно, что привязка разряда к катоду структурирована. В разряде формируется ярко светящийся канал, через который, как можно предположить, протекает основная часть тока. Канал начинает формироваться еще за несколько миллиметров до входа в трубочку. Видно, что канал заполняет лишь небольшую часть сечения трубочки. При выходе из трубочки канал распространяется до контакта с анодом. Из отдельных кадров видеозаписи были определены радиальные распределения интенсивности излучения вблизи входа и выхода трубочки. Распределения оказались осесимметричными и были обработаны с помощью обратного преобразования Абеля по методу [8], что позволило определить радиальные распределения коэффициента эмиссии плазмы $\varepsilon(r)$ в спектральном диапазоне, регистрируемом камерой, предполагая, что плазма является оптически тонкой. Примеры распределений $\varepsilon(r)$ показаны в правой части рис. 2. Полуширины распределений (ширины на половине высоты) $\Delta$ были использованы для оценки радиуса канала. Основная часть погрешности определения $\Delta$ определялась размером пиксела изображения и составляла $\sim 0.07 \mathrm{~mm}$. Величины полуширин, полученные в разных режимах, менялись в пределах $\Delta=0.35-0.45 \mathrm{~mm}$. Различие укладывается в пределы погрешности.

Если предположить, что ток переносится в основном внутри канала, диаметр которого $d=\Delta$, то плотность тока в нем при токах 0.1 и $0.3 \mathrm{~A}$ составляет соответственно $j \approx 100$ и $300 \mathrm{~A} / \mathrm{cm}^{2}$. Допустим, что дрейфовая скорость электронов порядка $10^{6} \mathrm{~cm} / \mathrm{s}$ [9] (учтена высокая концентрация водяного пара в плазме РЖНЭ [3]). Тогда концентрация электронов в канале исследуемого разряда $n \approx 6 \cdot 10^{14} \mathrm{~cm}^{-3}$ и $n \approx 2 \cdot 10^{15} \mathrm{~cm}^{-3}$ при токах 0.1 и 0.3 А соответственно.

Измерения потенциала плавающего зонда $U_{p}$ на оси разряда в различных режимах показали, что в той части разряда, в которой, согласно кадрам видеозаписи, формируется яркий токовый канал, потенциал меняется линейно, напряженность поля $E$ постоянна (рис. 2, левая часть). Результаты измерений $E$ в различных режимах показаны на рис. 3 . Видно, что с ростом тока $E$ уменьшается. При фиксированном токе увеличение расхода газа вызывает рост $E$.

Спектроскопические измерения показали, что, как и РЖНЭ в стационарной атмосфере $[2,4]$, разряд в потоке воздуха излучает в основном в фиолетовой и ультрафиолетовой областях спектра, однако есть и отличие. В спектре исследуемого разряда присутствуют линии атомарного водорода, интенсивность которых существенно выше шумов не только в прикатодной области, как это наблюдалось в [2,4], но и на всем протяжении разряда. Это можно объяснить тем, что с 


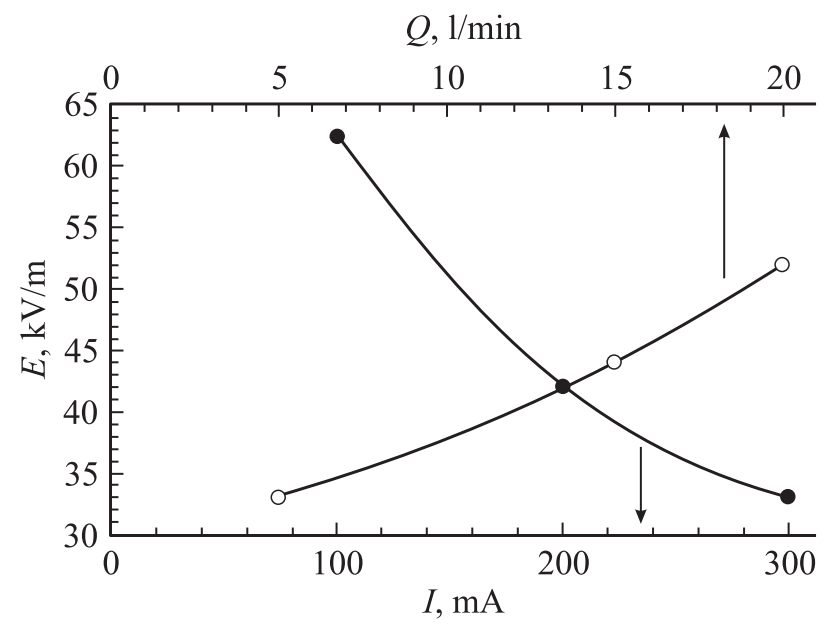

Рис. 3. Электрическое поле в токовом канале в разных режимах: • - зависимость $E$ от тока при постоянном $Q=51 / \mathrm{min}$, $\circ-$ зависимость $E$ от расхода при постоянном $I=0.3 \mathrm{~A}$.

одной стороны выделяющийся при электролизе воды водород захватывается потоком газа в канал разряда, с другой - что в этом разряде величина тока от 1.5 до 5 раз больше. Из отношения интенсивностей линий $H_{\alpha}$ и $H_{\beta}$ можно оценить температуру электронов $T_{e}$. Результаты оценок $T_{e}$ в различных режимах для значений $x \approx 0$ и $-7 \mathrm{~mm}$ приведены в таблице. Возможность использования относительных интенсивностей $H_{\alpha}$ и $H_{\beta}$ для оценки температуры электронов будет обоснована ниже. В настоящей работе мы ограничили спектроскопические измерения только измерениями линий атомарного водорода. Подробные измерения всего спектра в различных режимах будут выполнены в последующих исследованиях.

Измерение температуры газа с помощью термопары при $I=0.1 \mathrm{~A}, Q=5 \mathrm{l} / \mathrm{min}$ показало, что температура газа в бестоковой части струи могла быть измерена до сечения примерно на $2 \mathrm{~mm}$ выше анода (рис. 2) и составляла в этом сечении $T_{g} \approx 1450 \mathrm{~K}$. Измерениям ближе к аноду мешали помехи, вызванные пробоем на термопару. Экстраполируя зависимость $T_{g}(x)$, можно получить для несущей ток струи вблизи анода оценку: $T_{g} \approx 1700 \mathrm{~K}$. С увеличением тока $T_{g}$ в струе растет. Из-за пробоев при $I=0.2 \mathrm{~A}, Q=5 \mathrm{l} / \mathrm{min}$ измерения были и возможны лишь до сечения на $4 \mathrm{~mm}$ выше анода и дали значение $T_{g} \approx 1650 \mathrm{~K}$. При $I=0.3 \mathrm{~A}, Q=51 / \mathrm{min}$ измерения были возможны лишь до сечения на $6 \mathrm{~mm}$

Температура электронов в зависимости от тока при $Q=51 / \mathrm{min}$

\begin{tabular}{c|c|c}
\hline$I, \mathrm{~mA}$ & $\begin{array}{c}T_{e}, \mathrm{~K} \\
(x \approx-7 \mathrm{~mm})\end{array}$ & $\begin{array}{c}T_{e}, \mathrm{~K} \\
(x \approx 0 \mathrm{~mm})\end{array}$ \\
\hline 100 & 4500 & 5100 \\
200 & 4500 & 4900 \\
300 & 3600 & 4200
\end{tabular}

выше анода и дали $T_{g} \approx 1950 \mathrm{~K}$. Учитывая сильную нелинейность зависимостей $T_{g}(x)$, экстраполяция этих зависимостей на большие расстояния в сторону анода неразумна. Поэтому оценок $T_{g}$ в токовой части струи при токах более 0.1 А получить не удалось. Можно сравнить величины $T_{g}$ в бестоковой части струи в сечении примерно на $6 \mathrm{~mm}$ выше анода. Для токов 0.1, 0.2 и 0.3 А при расходе $Q=51 / \mathrm{min}$ они соответственно составляют: 900, 1450 и $1950 \mathrm{~K}$.

Для обоснования возможности оценки $T_{e}$ из относительных интенсивностей водородных линий при $I=0.1$ А будем использовать приведенные выше оценки концентрации электронов и концентрации нейтральной компоненты плазмы, полученные с учетом температуры газа. Используя эти значения, оценим отношение частот электрон-электронных столкновений и столкновений электронов с нейтралами: $v_{e e} / v_{e n} \sim 10^{-1} \gg \delta$ $(\delta$ - параметр, характеризующий передачу энергии от электронов к тяжелой компоненте с учетом коэффициента неупругих потерь). Оценка показывает, что функция распределения электронов по энергии близка к максвелловской [6]. Оценим также частоту неупругих столкновений электронов с атомами водорода $v_{e H}$. Предположим, что весь водород, выделяющийся при диссоциации воды в водяном катоде, подхватывается потоком воздуха в разряд, где значительная часть молекул водорода диссоциирует, и получим значение частоты столкновений $v_{e \mathrm{H}} \sim 10^{7} \gg 1 / \tau \sim 10^{5}$ ( $\tau-$ радиационное время жизни возбужденных уровней водорода). Оценка показывает, что заселенность уровней определяется столкновительными процессами. Различие энергий уровней, излучающих $H_{\alpha}$ и $H_{\beta}$, составляет $\sim 0.6 \mathrm{eV} \sim k T_{e}$. Все это подтверждает предположение, что температуру электронов можно получить из относительной интенсивности линий $H_{\alpha}$ и $H_{\beta}$.

В работе [10] приведены результаты расчета стационарного разряда внутри цилиндрического канала при атмосферном давлении воздуха в широком диапазоне токов $(I=0.01-1 \mathrm{~A})$. В расчете учитывались эффекты, обусловленные неравновесностью состояния плазмы. Расчеты сделаны для каналов различного радиуса $(R=1,3,10 \mathrm{~mm})$. Количественное сравнение расчетов $[10]$ с настоящим экспериментом невозможно, так как расчет сделан для стационарного случая, а эксперимент - для потока. Однако можно сравнить результаты на качественном уровне. В частности, напряженность поля в расчете составляет несколько десятков $\mathrm{kV} / \mathrm{m}$ и уменьшается при увеличении тока. Это находится в качественном согласии с результатами измерения поля, приведенными на рис. 3. Эффективный радиус разряда $R_{\text {eff, }}$ характеризующий радиус токового канала, при токах от 0.1 до 0.3 А согласно [10] при $R=1 \mathrm{~mm}$ монотонно возрастает от $R_{\text {eff }}=0.2$ до $0.3 \mathrm{~mm}$, а при $R=3 \mathrm{~mm}$ меняется немонотонно и составляет в среднем $R_{\text {eff }} \approx 0.45 \mathrm{~mm}$. Это находится в качественном согласии с полученной в эксперименте оценкой диаметра токового канала $d \approx 0.35-0.45 \mathrm{~mm}$. Соответственно и в 
расчете, и в эксперименте плотность тока $\sim 10^{2} \mathrm{~A} / \mathrm{cm}^{2}$. Отметим, что по данным экспериментальной работы [11], в которой изучался тлеющий разряд при атмосферном давлении, при токе разряда $10 \mathrm{~mA}$ плотность тока в положительном столбе составляет $105 \mathrm{~A} / \mathrm{cm}^{2}$.

\section{Заключение}

В разряде с катодом из водопроводной воды, горевшем в потоке воздуха при давлении, близком к атмосферному, определено электрическое поле $E$, оценена плотность тока $j$ и концентрация электронов $n_{e}$ в трубке, формирующей поток, определена температура электронов $T_{e}$, а также получена оценка температуры газа $T_{g}$. Измерения показали, что плазма в потоке находится в неравновесном состоянии. Несмотря на высокое давление температура тяжелых частиц существенно ниже температуры электронов. При $I=0.1 \mathrm{~A}$ отношение $T_{e} / T_{g} \approx 3$, но уменьшается с ростом тока. Представляет интерес температура заселения колебательных уровней, в первую очередь, молекулы азота. Для этой цели планируется провести спектроскопические измерения и моделирование спектра исследуемого разряда.

\section{Список литературы}

[1] Bruggeman P., Leys C. // J. Phys. D: Appl. Phys. 2009. Vol. 42. P. 053001.

[2] Andre P., Barinov Yu., Faure G., Kaplan V., Lefort A., Shkol'nik S., Vacher D. // J. Phys. D: Appl. Phys. 2001. Vol. 34. P. 3456-3465.

[3] Andre P., Aubreton J., Barinov Yu., Elchinger M.F., Fauchais P., Faure G., Kaplan V., Lefort A., Rat V., Shkol'nik S. // J. Phys. D: Appl. Phys. 2002. Vol. 35. P. $1846-1854$.

[4] Andre P., Barinov Yu., Faure G., Shkol'nik S.M. // J. Phys. D: Appl. Phys. 2011. Vol. 44. P. 375202.

[5] Andre P., Barinov Yu., Faure G., Shkol'nik S.M. // J. Phys. D: Appl. Phys. 2011. Vol. 44. P. 375203.

[6] Бенилов М.С. // ТВТ. 1988. Т. 26. Вып. 5. С. 993-1004.

[7] Баринов Ю.А., Школьник С.М. // ЖТФ. 2002. Т. 72. Вып. 3. C. $31-37$.

[8] Луизова Л.А. // Оптика и спектроскопия. 1982. № 4. Т. 52. C. 690-695.

[9] Хаксли Л., Кромптон Р. Диффузия и дрейф электронов в газах. М.: Мир, 1977. Гл. 14. С. 656-657.

[10] Benilov M.S., Naidis G.V. // J. Phys. D: Appl. Phys. 2003. Vol. 36. P. 1834-1841.

[11] Staack D., Farouk B., Gutsol A., Fridman A. // Plasma Sources Sci. Techn. 2005. Vol. 14. P. 700-711. 